\title{
A Modern Approach to the Treatment of Mitochondrial Disease
}

\author{
Sumit Parikh, MD, Russell Saneto, DO, PhD, Marni J. Falk, MD, Irina Anselm, MD, Bruce H. \\ Cohen, MD, Richard Haas, MB, BChir, MRCP, and The Mitochondrial Medicine Society

\section{Opinion statement}

The treatment of mitochondrial disease varies considerably. Most experts use a combination of vitamins, optimize patients' nutrition and general health, and prevent worsening of symptoms during times of illness and physiologic stress. We agree with this approach, and we agree that therapies using vitamins and cofactors have value, though there is debate about the choice of these agents and the doses prescribed. Despite the paucity of high-quality scientific evidence, these therapies are relatively harmless, may alleviate select clinical symptoms, and theoretically may offer a means of staving off disease progression. Like many other mitochondrial medicine physicians, we have observed significant (and at times life-altering) clinical responses to such pharmacologic interventions. However, it is not yet proven that these therapies truly alter the course of the disease, and some experts may choose not to use these medications at all. At present, the evidence of their effectiveness does not rise to the level required for universal use.

Based on our clinical experience and judgment, however, we agree that a therapeutic trial of coenzyme Q10, along with other antioxidants, should be attempted. Although individual specialists differ as to the exact drug cocktail, a common approach involves combinations of antioxidants that may have a synergistic effect. Because almost all relevant therapies are classified as medical foods or over-the-counter supplements, most physicians also attempt to balance the apparent clinical benefit of mitochondrial cocktails with the cost burden that these supplements pose for the family.

\section{Introduction}

Treatment of mitochondrial disease is still in its infancy. Aside from symptom-based management, treatment of mitochon-drial disease focuses on maintaining optimal health, using preventive measures to mitigate symptom worsening during times of physiologic stress (such as infection, dehydration, or surgery), and avoiding mitochondrial toxins.

Some evidence supports the use of antioxidant supplements aimed at reducing reactive oxygen species that are produced in increased amounts in this disease. The evidence for these and other treatments is still developing. Progress has been slow for several reasons: 1) Mitochondria have been linked to human disease only since the 1960s, and much of the knowledge has been discovered in the past 20 years. 2) Mitochondrial diseases are classified as rare disorders, so funding for research is limited when compared with more common ailments. 3) Similarly, because most treatments for mitochondrial diseases are categorized as medical foods, the financial incentive to study and develop these compounds is small. 4)

(C) 2009 by Current Medicine Group LLC

Corresponding author: Sumit Parikh, MD, Neurometabolism \& Neurogenetics, Cleveland Clinic, 9500 Euclid Avenue, S71, Cleveland, OH 44195, USA. sumit.parikh@gmail.com.

Disclosure: Dr. Parikh is a consultant for Solace Pharmaceuticals, a manufacturer of CoQ10 and creatine. Dr. Cohen is a member of the scientific advisory board and on the speaker's bureau for Transgenomic, Inc., a company that performs genetic testing. No other potential conflicts of interest relevant to this article were reported. 
Obtaining a standardized pool of patients for trials is difficult because of continually evolving diagnostic criteria, limited ability to establish molecular genetics-based diagnosis, fluctuations in symptom severity, and an overall lack of genotype-phenotype correlations.

Scientific support for the use of vitamin-based and cofactor-based mitochondrial therapies is accumulating. Such pharmacologic supplements are intended to promote critical enzymatic reactions, reduce putative sequelae of excess free radicals, and scavenge toxic acyl coenzyme A (acyl CoA) molecules, which accumulate in mitochondrial disease. Some supplements also may act as alternative energy fuels or may bypass biochemical blocks within the respiratory chain, although these mechanisms are more widely debated. Exercise also has an important role in mitochondrial disease therapy, as it has been shown to reduce the burden of unhealthy mitochondria; increase the percentage of healthy, nonmutated mitochondrial DNA (mtDNA); and improve endurance and muscle function.

Current clinical goals for mitochondrial disease therapy are to increase energy production in the form of adenosine triphosphate (ATP) and reduce free radical production in an effort to improve, or at least stabilize, disease signs and symptoms. Among mitochondrial disease experts, there is anecdotal evidence of such improvement. A variety of scientific studies have also demonstrated some clinical improvement, although the vast majority of studies evaluating antioxidant efficacy in mitochondrial disease have been short-term, nonrandomized trials.

No definitive means of halting disease progression is yet available, and the clinical ability to predict therapeutic responsiveness, achieve optimal dosing of medications, and quantify benefits in individual patients remains limited. The long-term benefits of treatment remain unproven.

Although the diagnosis of the myriad of mitochondrial diseases may be complicated, its discussion is limited here in order to focus on treatment. Readers are referred to detailed reviews of standard diagnostic approaches for both generalists and specialists in the December 2007 issue of Pediatrics [1] and the May 2008 issue of Molecular Genetics and Metabolism [2]. Formal diagnostic criteria can be reviewed in a 2006 issue of Neurology [3].

\section{Treatment}

\section{Diet and lifestyle}

Healthful nutrition-Secondary mitochondrial dysfunction occurs with extreme malnutrition, including anorexia, starvation, and illness-related cachexia [4, Class III].

Mitochondrial disease patients can have altered caloric needs compared with the general population. Optimizing the number and quality of calories has been shown to improve mitochondrial health in these patients [5•, Class III]. Evaluation of resting metabolic rate may aid in establishing the ideal caloric intake needed for the patient. Though specific dietary restrictions or changes are not universally recommended, a comprehensive evaluation of a patient's nutrition and potential deficiencies is needed. Some patients may need restriction of carbohydrate, protein, or fat. Caloric supplementation, enteral feeding, limited fasting, increased meal frequency, and intravenous nutrition are all potential therapeutic avenues to consider.

Treating swallowing dysfunction, abnormal gut motility, behavioral feeding issues, and gastroesophageal reflux is recommended to allow for optimal nutritional intake. 
Immunizations-There is no scientific evidence that immunizations exacerbate mitochondrial disease manifestations, and most mitochondrial disease specialists strongly advocate for immunizations in an effort to protect against potentially devastating infectious diseases, which may be life-threatening for the mitochondrial disease patient.

Immunizations may be administered individually or in limited batches to reduce metabolic stress. Though there is a lack of evidence to support this practice, such an option may be an acceptable alternative for parents and patients with concerns about immunizations, avoiding the risk of forgoing immunizations altogether.

A related report has focused on whether immunizations in children who appear healthy but who have a latent, predisposing mitochondrial condition may bring out autism-spectrum behaviors [6, Class IV].

Avoiding mitochondrial toxins-Many medications and environmental toxins impair mitochondrial functions. The mechanisms of action vary with individual drugs. They include direct inhibition of the electron transport chain, increased creation of reactive oxygen species, impaired mitochondrial protein transport, inhibition of mitochondrial DNA replication, or some combination of these mechanisms.

Table 1 lists drugs that are known to cause symptoms in vivo. Many other mitochondrial toxins are known to decrease mitochondrial function in vitro.

Ketogenic diet-The ketogenic diet is a high-fat diet that effectively treats some forms of medically refractory epilepsy [7,8, Class I]. Recent animal research has suggested that the ketogenic diet may be beneficial in optimizing mitochondrial function [9, Class III].

Because many mitochondrial disease patients have secondary fatty acid oxidation disorders, there are limited data on use and safety of the ketogenic diet in patients with these conditions. Only a single report has looked at the lack of efficacy of the ketogenic diet in children with electron transport chain defects and intractable seizures [10, Class IV].

The ketogenic diet is the standard of care for pyruvate dehydrogenase deficiency, but it is contraindicated in patients with known fatty acid oxidation disorders and pyruvate carboxylase deficiency.

\section{Pharmacologic treatment}

- Table 2 lists the medications most commonly used by mitochondrial medicine physicians to treat mitochondrial disease. Some of these medications are discussed in further detail below.

Coenzyme Q10-The evidence supporting the use of coenzyme Q10 (CoQ10, also known as ubiquinone) in mitochondrial disease was reviewed in 2007 [11••, Class IV]. CoQ10 is endogenously synthesized in mammalian mitochondria and is an integral component of the mitochondrial electron transport chain, shuttling electrons from complexes I or II and a number of other electron donors, including electron transfer factor, which moves electrons from fatty acid beta oxidation.

CoQ10 is found in all cell and organelle membranes, where it can participate in redox shuttling. It has an important intracellular signaling role, as well as both antioxidant and prooxidant roles. CoQ10 modulates the mitochondrial permeability transition pore involved in apoptosis and activates uncoupling proteins. 
CoQ10 biosynthetic defects underlie several different phenotypes of human mitochondrial disease. These include neonatal encephalopathy with nephropathy (COQ2) [12, Class IV]; Leigh syndrome, lactic acidosis, and nephropathy (PDSS2) [13, Class IV]; infantile nephropathy, hepatopathy, retardation (PDSS1) [14, Class IV]; and recessive ataxia, cerebellar atrophy \pm retardation, lactic acidosis, and exercise intolerance (ADCK3) [15, Class IV]. These disorders, which may respond to exogenous CoQ10 administration, represent an important group of treatable mitochondrial diseases.

Pharmacokinetics: CoQ10 is insoluble in water. Powder formulations of CoQ10 have very poor intestinal absorption. No increase in CoQ10 plasma levels was achieved when 3000 $\mathrm{mg} / \mathrm{d}$ was compared with administration of $2400 \mathrm{mg} / \mathrm{d}$, suggesting a block to gastrointestinal absorption above $2400 \mathrm{mg}$ in adults [16, Class IV]. Improved bioavailability has been seen with the use of nano-particles in suspension [17].

Recently, reduced CoQ has become commercially available in the form of ubiquinol. This formulation is three to five times better absorbed when compared with the oxidized form of CoQ, ubiquinone.

CoQ is carried in the blood in white cells and platelets. In plasma, CoQ is largely (95\%) bound to lipoproteins in the reduced ubiquinol form.

The plasma half-life of administered CoQ10 is about 36 hours. Following 1 month of oral administration of two relatively bioavailable CoQ10 formulations (a liquid and a wafer), blood levels returned to baseline 2 weeks following CoQ10 cessation (Haas, unpublished data, Class IV).

Catabolic pathways for CoQ have not yet been characterized.

CoQ levels in blood and tissues fall with normal aging. CoQ levels in a 70-year-old are about $50 \%$ of those in a 20 -year-old.

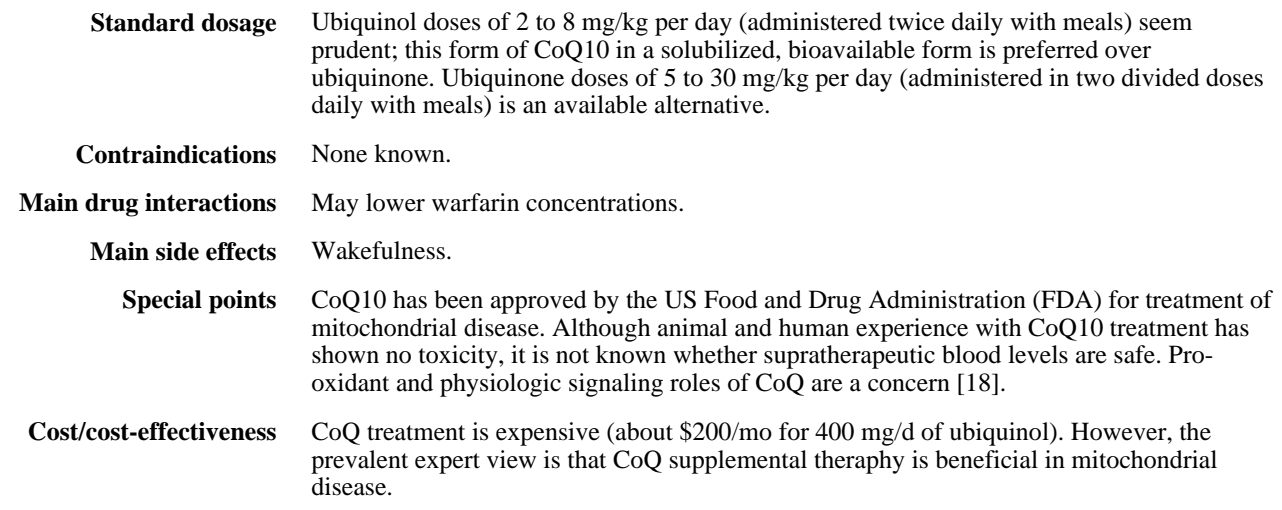

Riboflavin-Riboflavin is a water-soluble B vitamin $\left(\mathrm{B}_{2}\right)$ that serves as a flavoprotein precursor. It is a key building block in complex I and II and a cofactor in several other key enzymatic reactions involving fatty acid oxidation and the Krebs cycle.

Multiple acyl CoA dehydrogenase deficiency (MADD), typically caused by electrontransport flavoprotein dehydrogenase $(E T F D H)$ gene mutations, is a known inborn error of metabolism involving several of these enzymatic reactions; riboflavin supplementation using moderate to high doses can lead to amelioration of symptoms and slowing of disease

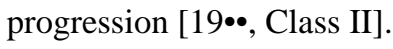


Several non-randomized studies have shown riboflavin to be efficacious in treating mitochondrial diseases, specifically complex I and/or complex II disease [20-22, Class IIIIV].

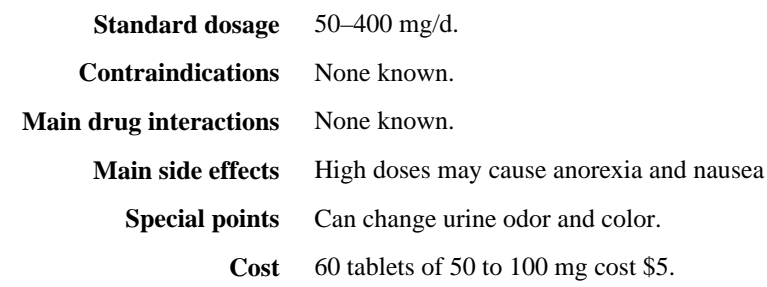

L-cretine-Creatine, a compound present in cells, combines with phosphate in the mitochondria to form phosphocreatine. It serves as a source of high-energy phosphate, released during anaerobic metabolism. It also acts as an intracellular buffer for ATP and as an energy shuttle for the movement of high-energy phosphates from mitochondrial sites of production to cytoplasmic sites of utilization.

The highest concentrations of creatine are found in tissues with high energy demands, such as skeletal muscle and brain. Creatine is continuously replaced through a combination of diet and endogenous synthesis.

A reduction in phosphocreatine in skeletal muscle in patients with mitochondrial myopathies has been reported by direct measurement [23, Class IV]. Patients with mitochondrial encephalomyopathies may also have a reduction in brain creatine [24, Class IV].

A randomized, controlled trial in adult patients with mitochondrial cytopathies showed benefits from creatine taken as an initial dose of $5 \mathrm{~g}$ twice a day for 2 weeks followed by $2 \mathrm{~g}$ twice a day for 1 week [25, Class II]. Treatment resulted in an increase in high-intensity, isometric, anaerobic, and aerobic power. No effects were observed on aerobic cycleergometry exercise variables, body composition, 2-minute walk, or activity of daily living scores. Similar results were reported in four pediatric patients with mitochondrial encephalomyopathies [26, Class IV]. Other studies have failed to show benefit of creatine treatment in mitochondrial disease.

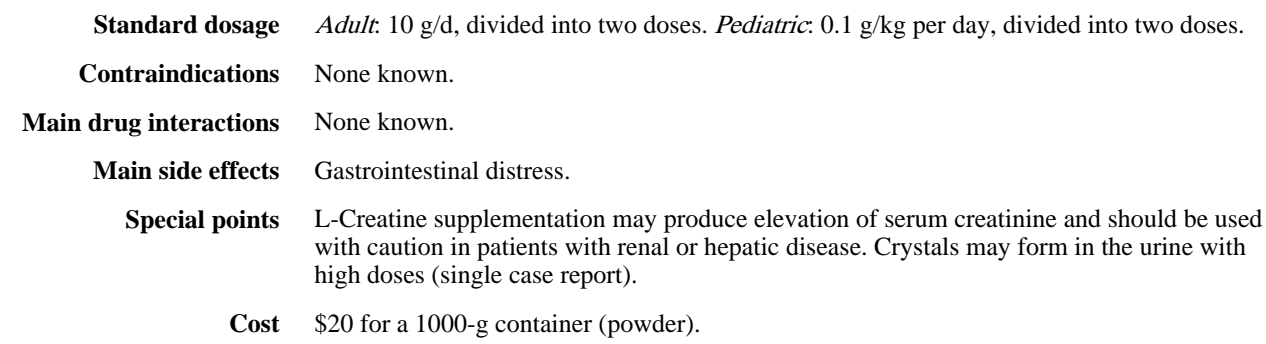

L-Arginine-Arginine is a semi-essential amino acid involved in growth, urea detoxification, and creatine synthesis. L-arginine produces nitric oxide, which has neurotransmitter and vasodilatory properties.

An initial small study demonstrated that intravenous (IV) administration of L-arginine (500 $\mathrm{mg} / \mathrm{kg} / \mathrm{dose}$ ) quickly decreased the severity of strokelike symptoms, enhanced the dynamics of microcirculation, and reduced tissue injury from ischemia in patients with mitochondrial encephalomyopathy, lactic acidosis, and strokelike episodes (MELAS) [27, Class III]. In a larger study, a decrease in clinical severity and frequency of strokelike events was 
demonstrated in MELAS patients treated prophylactically with oral L-arginine (150-300 $\mathrm{mg} / \mathrm{kg} / \mathrm{d}$ ) [28, Class III]. Responsive individuals tended to have baseline plasma arginine levels in the lower limits of normal.

We have successfully used L-arginine for metabolic strokes in patients with MELAS and other types of mitochondrial disease, both as an IV medication in the acute setting and as a daily oral medication to reduce the frequency of events.

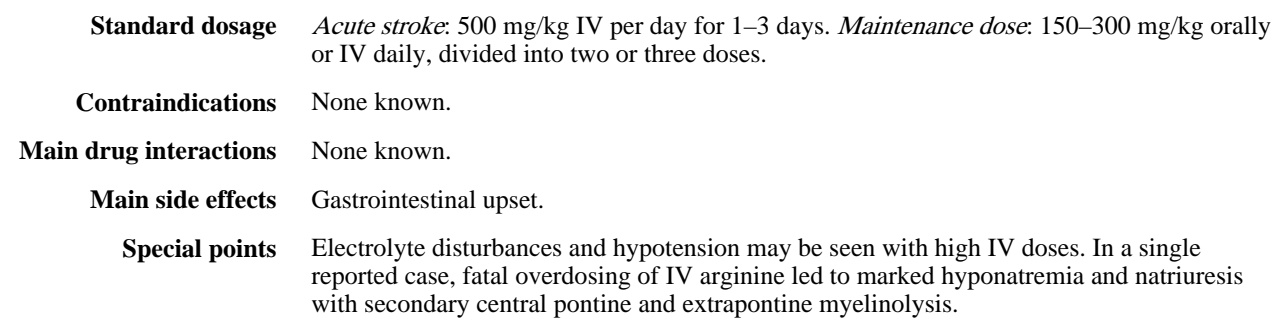

L-Carnitine-L-Carnitine is a cellular compound that plays a critical role in the process of mitochondrial $\beta$-oxidation of fatty acids and the esterification of free fatty acids that may otherwise be sequestered by CoA. Carnitine transfers long-chain fatty acids across the mitochondrial inner membrane as acylcarnitine esters. These esters are oxidized to acetyl CoA, which enters the Krebs cycle and results in subsequent generation of ATP via oxidative phosphorylation.

Certain tissues, such as skeletal muscle, heart, and liver, largely depend on $\beta$-oxidation for ATP production. Carnitine may prevent CoA depletion and remove excess, potentially toxic, acyl compounds; at this time there is no pharmacologic method to increase CoA levels.

Diet is the source of $75 \%$ of carnitine; $25 \%$ is synthesized in the body, predominantly within muscle, liver, and kidneys. Skeletal muscles contain 90\% of total body carnitine [29].

Plasma concentration of carnitine is regulated by its active reabsorption in the proximal renal tubules.

Primary carnitine deficiency due to defective carnitine synthesis or transport is not a typical feature of mitochondrial disorders. However, patients with respiratory chain defects tend to have lower than average free carnitine levels in plasma and increased esterified carnitine levels. This shift may reflect partial $\beta$-oxidation impairment [30••, Class IV]. L-carnitine supplementation for mitochondrial disorders is a common practice aimed at restoring free carnitine levels and removing accumulating toxic acyl compounds.

Carnitine is available as a generic or brand-name prescription (Carnitor; Sigma-Tau Pharmaceuticals, Gaithersburg, MD) for oral or IV use in mitochondrial disease. It is commonly used empirically in combination with other vitamins and cofactors (Table 2) [31, Class IV]. No studies have confirmed a benefit of isolated use of carnitine in patients with primary mitochondrial disorders.

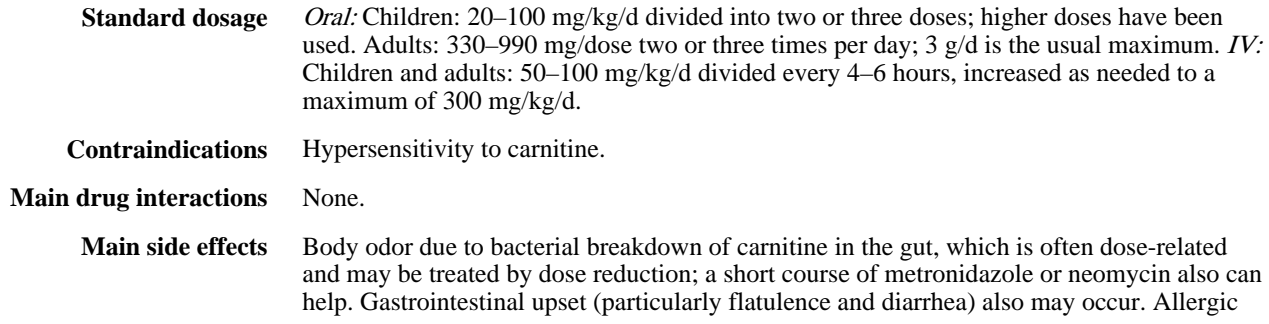




\section{reactions are rare. Tachyarrhythmia occurred in an animal model of very long chain acyl CoA dehydrogenase def ciency. Chronic administration of high doses of oral carnitine in patients with severely compromised renal function may result in accumulation of potentially toxic metabolites [32, Class IV]. \\ Cost \$70: 90 generic tablets (330 mg). \$35: $118 \mathrm{~mL}$ of $1 \mathrm{~g} / 10 \mathrm{~mL}$ generic suspension.}

Other redox agents-Thiamine $\left(\mathrm{B}_{1}\right)$, vitamins $\mathrm{C}$ and $\mathrm{E}$, and alpha-lipoic acid also have been used in mitochondrial disease patients, individually or as part of an antioxidant cocktail. These therapies have less scientific data available regarding treatment of mitochondrial disease than the medications discussed here in more detail.

Folinic acid-Folinic acid is a reduced form of folic acid, a water-soluble B vitamin ( $\left.\mathrm{B}_{9}\right)$ involved as a cofactor in multiple metabolic reactions. It is most commonly available as leucovorin, containing the $\mathrm{D}$ (inactive) and $\mathrm{L}$ (active) isomers of the chemical. It is also available as Deplin (5-methyl-tetrahydrofolate, 5-MTHF; PamLab, Mandeville, LA), which is the natural transport form of folate across the blood-brain barrier, and Isovorin (levofolinic acid; Spectrum Pharmaceuticals, Irvine, CA), containing only the L-isomer (active) form.

A few case reports [33, Class IV] and one small case series [34, Class IV] have suggested that mitochondrial disease may lead to secondary cerebral folate deficiency, defined by low cerebrospinal fluid (CSF) folate, especially of the $\mathrm{C} 1$ donor, 5-MTHF. The mechanism of cerebral folate deficiency in mitochondrial disease is unclear; it is postulated to result from a failure to produce adequate ATP for active folate transport across the blood-brain barrier.

Most cases of proven 5-MTHF CSF deficiency in mitochondrial disease have occurred in patients with Kearns-Sayre syndrome. Studies in other forms of mitochondrial disease have been less consistent in demonstrating cerebral folate deficiency.

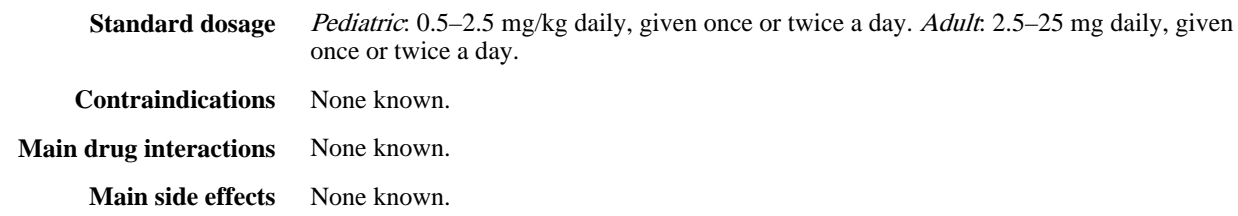

\section{Historical treatments now less commonly used}

Dichloroacetate-Dichloroacetate (DCA) is a potent lactate-lowering drug. It activates the pyruvate dehydrogenase complex by inhibiting the activity of pyruvate dehydrogenase kinase, which normally phosphorylates and inhibits the enzyme. The ability of DCA to keep the pyruvate dehydrogenase complex in an active state reduces the accumulation of lactate in body tissues.

Since its discovery in 1973, DCA has been used to treat conditions associated with lactic acidosis, including mitochondrial disorders. Most published reports about the use of DCA are individual case reports. Several controlled trials have not led to conclusive evidence of the benefits of DCA in patients with mitochondrial disorders [35, Class II].

An open-label trial of DCA used for up to 7 years in patients with mitochondrial disorders demonstrated a variable response, consistent with the genetic heterogeneity and the intermittent nature of these disorders. The study's conclusion was that DCA may provide at least temporary benefit for some patients with mitochondrial disorders [36, Class III]. 
A randomized double-blind, placebo-controlled, 3-year crossover trial of DCA in patients with MELAS had to be terminated because of DCA toxicity, consisting of peripheral neuropathy that overshadowed the assessment of potential benefit [37, Class II].

DCA has "orphan drug" status. It is used on a compassionate basis or as a part of research protocols in several centers in the United States. Its use declined after the publication of the results from the MELAS trial.

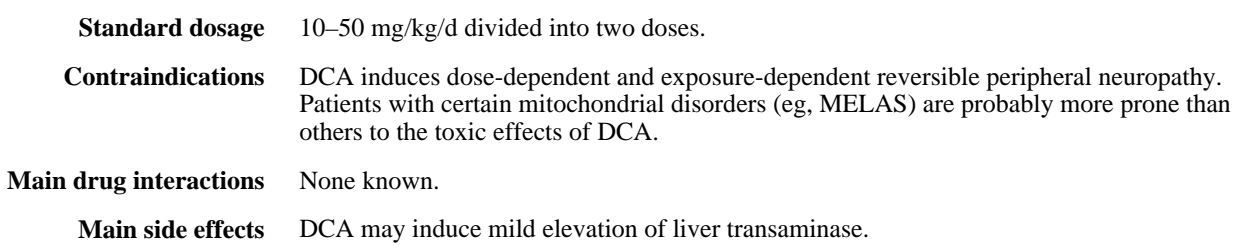

Succinate and menadione-Succinate may be used as an anaplerotic compound in specific patients.

Menadione has been banned by the FDA because of toxicity, including hemolytic anemia in glucose-6-phosphate dehydrogenase (G6PD) deficiency and neonatal hepatopathy.

\section{Interventional procedures}

For most patients with mitochondrial disease, typical acute interventions are limited to preventing worsening of the disease and symptoms. Some individuals with metabolic and mitochondrial diseases are more sensitive to physiologic stressors such as minor illness, dehydration, fever, temperature extremes, surgery, anesthesia, and prolonged fasting or starvation. During such stress, rapid systemic decompensation may occur. Preventive measures are aimed at avoiding (or at least not exacerbating) such decompensation.

Mainstays of treatment during or prior to acute metabolic decompensation in mitochondrial disease include keeping patients well hydrated, providing sufficient anabolic substrate, correcting secondary metabolic derangements, avoiding pharmacologic mitochondrial toxins, and providing cofactor and/or salvage therapies.

A supplementary table available online at the website of The Mitochondrial Medicine Society (http://mitosoc.org/blogs/diagnosis/illness-management) lists the basic assessment and management steps to take during times of catabolic stress. Pertinent details are noted below.

Intravenous fluids and substrate therapy-No single protocol is optimal for every mitochondrial disease patient. Dextrose/electrolyte therapy should be considered if a patient is unable to maintain oral fluid intake in the face of a catabolic stressor, including fever, illness, or vomiting. Any underlying infection and fever should be aggressively treated [38, Class III].

Hospital admission should be considered, not exclusively for dehydration, but to prevent catabolism by providing an anabolic food in the form of dextrose. To prevent catabolism, total parenteral nutrition should be offered at an early stage. The use of intravenous lipids may be limited because of the frequent occurrence of impaired fatty acid oxidation in mitochondrial disease.

Assessment of the patient's cardiac and renal status must be performed prior to aggressive fluid therapy. Hydration and substrate therapy involves providing 5\% or 10\% dextrose- 
containing IV fluids, given at 1.25 to 1.5 times the maintenance rate. IV fluids should not contain lactated Ringer's solution. Fluids should be weaned based on laboratory parameters, oral intake, and resolution of the underlying metabolic stressor.

If the patient is acutely acidotic $(\mathrm{pH}<7.22$ or bicarbonate level $<14 \mathrm{mM})$, metabolic acidosis can be controlled by administering sodium bicarbonate as a bolus $(1 \mathrm{mEq} / \mathrm{kg})$ followed by a continuous infusion.

Hyperammonemia can occur because of secondary inhibition of the urea cycle. As treatment for the metabolic decompensation proceeds, the ammonia level should diminish. A level less than $200 \mu \mathrm{M}$ may require salvage therapy or dialysis.

A high dextrose delivery with D10 or D20 may be needed, especially if fluids containing 5\% dextrose are not correcting acidosis or metabolic derangements. When higher dextrose delivery is given, insulin may also be required. Insulin controls hyperglycemia and serves as a potent anabolic hormone, promoting protein and lipid synthesis, removal of plasma free fatty acids, and improved adenosine translocase activity. Insulin is typically given in the intensive care unit, with the initial dose in the range of 0.05 to $0.1 \mathrm{U} / \mathrm{kg}$ per hour, and is titrated accordingly.

Once the initial crisis passes, enteral feeding should be considered. Protein, lipids, or both can be added if there are no concomitant disorders of protein or fat catabolism. Once the patient's laboratory parameters begin to normalize, restarting the patient's home-based diet is advised.

Antioxidant therapy during acute illness-L-Carnitine therapy during an acute illness may be beneficial. It should be given intravenously at a dosage of at least $100 \mathrm{mg} / \mathrm{kg}$ per day. Doses of up to $300 \mathrm{mg} / \mathrm{kg}$ per day have been used. If the patient has been taking a higher oral dose, that dose should be used intravenously for treatment.

CoQ10 and other antioxidants such as vitamin $\mathrm{C}$ and $\mathrm{E}$ are also given at the doses listed in Table 2. Any other supplements and antioxidants being given at home should be continued by mouth if possible. There is no intravenous formulation of CoQ10.

IV L-arginine therapy should be considered for a patient with a possible metabolic stroke.

Medication contraindications-Individuals with mitochondrial disease generally should avoid valproic acid, statins, aminoglycoside antibiotics, and erythromycin. There are no absolute contraindications, however, and these medications can be given if no appropriate alternative is available, as long as a prior adverse reaction to the medication has not occurred.

If a medication such as valproate is to be used for the first time during an acute illness, liver enzymes, ammonia, and synthetic liver function should be closely monitored.

Anesthesia-Questions remain about anesthetic sensitivity in mitochondrial disease patients. Some individuals with mitochondrial metabolic diseases are more sensitive to volatile anesthetics and need a much lower dose to achieve a bispectral index (BIS) value less than 60. This effect has been seen more often in patients with reduced complex I capacity. Sevof urane may be better tolerated than isoflurane or halothane [39, Class IV].

The potential risk of propofol in mitochondrial disease patients is debated, but propofol has been used routinely in many of these patients for brief periods of sedation (less than 30-60 
minutes) without apparent clinical problems. Limiting propofol use to short procedures and brief periods of sedation is currently advised.

\section{Surgery}

- During preoperative and postoperative fasting, catabolism can be prevented by using dextrose-containing IV fluids, which should not contain lactated Ringer's solution. Routine chemistries, a complete blood count, liver function (synthetic and cellular), ammonia, glucose, ketosis, and lactic acidosis should be monitored and any derangements should be corrected. IV fluids are continued until the time of discharge, because they are intended to deter catabolism and not simply to treat dehydration.

Vagus nerve stimulation-Children with mitochondrial disease often have seizures that are not responsive to antiepileptic drugs. Therapy using vagus nerve stimulation (VNS) has been reported only in a single small series $(n=5)$ of children with electron transport chain dysfunction. Duration of VNS and seizure frequency was followed over a period of 3.4 to 10.75 years. None of the children had reduced seizure activity with VNS placement [40, Class IV]. There is also anecdotal evidence among the coauthors of this article that VNS devices have been unsuccessful in decreasing epilepsy in mitochondrial disease patients.

Organ transplantation-Most mitochondrial diseases involve multiple organ systems, so the transplantation of single organs is not therapeutically appropriate, but there are uncommon circumstances in which specific organ transplantation may be considered. There is also some evidence that patients with electron transport chain deficiencies may be candidates for liver transplants, given the correct clinical circumstances (mostly in children with isolated liver involvement and normal neurologic examinations). Long-term data and outcomes are lacking.

Some of the authors have personal experience with beneficial effects of cardiac transplantation in cardiomyopathy due to mitochondrial disease. Some of these patients have been observed for 5 years.

Two specific mitochondrial depletion syndromes, one involving the liver and the other involving gastrointestinal function, may represent exceptions to the rule. Patients having the hepatocerebral syndrome produced by mutations in deoxyguanosine kinase are currently considered for liver transplantation, which has led to long-term survival in some cases with a lack of neurologic involvement. Liver transplantation in patients with neurologic disease was not associated with improved survival [41, Class IV].

The second depletion syndrome, mitochondrial neurogastrointestinal encephalopathy (MNGIE), may be the single mitochondrial disorder for which stem cell transplantation may replete the missing enzyme activity and result in long-term clinical improvement. MNGIE is a disorder of nucleotide utilization that results in severe gastrointestinal malabsorption, encephalopathy, leukodystrophy, and myopathy. The compromised enzyme activity of thymidine phosphorylase increases the pool of deoxythymidine triphosphate and produces an imbalance in the purine pools, thereby altering mitochondrial DNA replication and repair. Enzyme replacement using platelets or other hematopoietic cells has provided limited disease treatment. Stem cell transplantation has proven to be highly efficacious, both in symptom relief and in duration of treatment effects [42, Class III]. The number of patients who have received stem cell transplants is limited, but the results in these few have been extremely encouraging, with the longest-living survivor now more than 40 months past transplantation and making a good recovery (Hirano, personal communication). 


\section{Assistive devices}

Cochlear implants-Sensorineural hearing loss occurs in many mitochondrial disorders, most notably in association with specific mtDNA mutations. The A1555G mutation is seen in individuals who have extreme susceptibility to hearing loss upon aminoglycoside exposure. Spontaneous hearing loss is associated with the A3243G mutation in MELAS and the mtDNA deletions seen in Kearns-Sayre syndrome. Sensorineural hearing loss also can be seen in other nuclear and mitochondrial DNA disorders.

Cochlear implants consist of an external component, which includes a microphone and speech processor with a radiofrequency transducer, and an intracranial portion consisting of another coil (positioned beneath the radiofrequency coil) connected to electrodes placed within the cochlea of the inner ear. Following surgical implantation, the patient and therapist adjust the speech processor to best suit the person's specific pattern of hearing loss, and the additional auditory information can be useful to regain some degree of hearing. Efficacy depends on the degree of hearing loss and the individual's premorbid ability to process auditory information. In some patients, the device allows only the detection of environmental sounds, such as a doorbell or the presence of speech, whereas it allows other patients to decipher language in a quiet environment [43, Class II].

In one study of 12 patients with profound postlingual hearing loss associated with mitochondrial disease ( 10 with $>90 \mathrm{~dB}$ hearing loss over the frequencies used for speech), 7 regained the ability to use the telephone and 10 regained useful speech recognition [44, Class IV].

An MRI cannot be obtained after receiving some models of cochlear implants.

The cost of the procedure (including evaluation, hardware, surgical and hospital fees, and rehabilitation) ranges from $\$ 45,000$ to $\$ 105,000$, with an average of about $\$ 60,000$ per ear.

Cardiac pacemakers and defibrillators-Cardiac conduction defects with or without associated cardiomyopathy are part of the spectrum of illness in some mitochondrial disorders, most notably in patients with Kearns-Sayre syndrome. Cardiac manifestations may also occur in patients with mtDNA disorders, fatty acid oxidation defects, and disorders due to nuclear genes, such as seen in Friedreich's ataxia and Barth syndrome [45, Class III].

The newer automatic internal cardiac defibrillators (AICDs) are implant-able medical devices that monitor the cardiac rhythm, function as a pacemaker if necessary, can determine whether or not a rhythm disturbance is pathogenic, and override the pacing to perform a synchronized cardioversion or an unsynchronized shock in the case of ventricular defibrillation. Less sophisticated external devices are available, often as a bridge to AICD.

Limited literature (mainly case reports and small, uncontrolled series) supports some efficacy, so the use of an AICD may be considered for patients with mitochondrial cardiac conduction defects with or without cardiomyopathy. AICD success in other disease states adds support for their consideration in the population of patients with mitochondrial cardiac conduction disorders [46, Class IV].

The cost of the procedure (including evaluation, hardware, surgical and hospital fees, and 4 years of follow-up) is estimated to be $\$ 98,000$ (1997 data). Newer data suggest that the device itself costs about $\$ 28,000$, with a cost of $\$ 34,000$ to $\$ 74,000$ per quality-adjusted life year [47, Class II]. 


\section{Physical/speech therapy and exercise}

Exercise-Exercise is one of the few proven methods for improving mitochondrial functioning and decreasing the burden of unhealthy mitochondria [48, Class III]. Patients with mitochondrial cytopathy may have a low maximal oxygen uptake (peak $\mathrm{VO}_{2}$ ), which can lead to exercise intolerance, even with activities of daily living [49, Class III].

Whenever possible, exercise regimens should be supervised by a therapist, trainer, or kinesiologist. A 12-lead electrocardiogram should be obtained prior to beginning an exercise regimen. Patients should start at a very low intensity and brief duration and should progress gradually. Use of a recumbent exercise bicycle and/or pool therapy may be better tolerated than other regimens in those with gait instability or excessive fatigue. A simple carbohydrate-containing drink or meal prior to exercising may increase endurance.

Graded endurance exercise can improve exercise tolerance as well as biochemical enzyme activity and mutation burden [50•, Class III]. Resistance exercise (using weights) can be performed by many patients and can increase strength.

The exercise regimen should be halted during an illness or fasting state.

Therapies-The goal of therapy is not to change the underlying mitochondrial disease, but to preserve and maximize strength, mobility, and functioning. In addition to the more widely known physical, occupational, and speech therapies, respiratory therapy and hippotherapy should also be considered [51, Class III].

\section{Other treatments}

Hyperbaric oxygen-There is no evidence that hyperbaric oxygen therapy is beneficial for patients with mitochondrial disease, and there is a theoretical concern about oxygen toxicity in these patients.

\section{Emerging therapies}

Sirtuins-Sirtuin compounds (SIRT 1-7) modulate histone deacetylases (at lysine residues), which affect the activity of multiple metabolic enzymes via stimulation of the peroxisome proliferator-activated receptor (PPAR) family, the PPAR- $\gamma$ coactivator $1 a$ (PGC-1a), and insulin signaling pathway. PPAR signaling regulates gene expression of multiple metabolic pathways, including gluconeogenesis, metabolism of fatty acids, adipocyte differentiation, cell survival, and ubiquitination [52, Class II].

A known pharmacologic PPAR pan-agonist (bezafibrate), which is FDA-approved for diabetes, was recently demonstrated to improve lifespan and ameliorate muscle disease manifestations by increasing mitochondrial biogenesis and tissue ATP levels in a skeletal muscle conditional knockout mouse [53, Class IV].

SIRT-1 agonists are currently being developed for a variety of conditions, including mitochondrial disease, and are currently entering phase 2 clinical trials. No clinical trials have been completed to guide their use or determine their risks and benefits in primary mitochondrial disease.

Resveratrol-Resveratrol, an agent found in red wine, is an antioxidant, an apoptosis inhibitor, and an SIRT-1 agonist. Resveratrol has been studied for its potential role in the treatment of diabetes, cardiovascular disease, neurodegenerative disease, cancer, obesity, and aging [54, Class IV]. No clinical trials have been completed to guide its use or determine its risks and benefits in primary mitochondrial disease. 


\section{References and Recommended Reading}

Papers of particular interest, published recently, have been highlighted as:

- Of importance

$\bullet$ Of major importance

1. Haas RH, Parikh S, Falk MJ, et al. Mitochondrial disease: a practical approach for primary care physicians. Pediatrics. 2007; 120:1326-1333. [PubMed: 18055683]

2. Haas RH, Parikh S, Falk MJ, et al. The in-depth evaluation of suspected mitochondrial disease. Mol Genet Metab. 2008; 94:16-37. [PubMed: 18243024]

3. Morava E, van den Heuvel L, Hol F, et al. Mitochondrial disease criteria: diagnostic applications in children. Neurology. 2006; 67:1823-1826. [PubMed: 17130416]

4. Wortmann SB, Zweers-van Essen H, Rodenburg RJ. Mitochondrial energy production correlates with the age-related BMI. Pediatr Res. 2009; 65:103-108. [PubMed: 19096353]

5•. Morava E, Rodenburg R, van Essen HZ, et al. Dietary intervention and oxidative phosphorylation capacity. J Inherit Metab Dis. 2006; 29:589. The Nijmegen group reviews the role of malnutrition leading to oxidative phosphorylation abnormalities and normalization of these findings by renourishment. They present a small cohort of their patients as examples. [PubMed: 16786255]

6. Cave SF. The history of vaccinations in the light of the autism epidemic. Altern Ther Health Med. 2008; 14:54-57. [PubMed: 19043939]

7. Freeman JM, Vining EP, Pillas DJ, et al. The efficacy of the ketogenic diet-1998: a prospective evaluation of intervention in 150 children. Pediatrics. 1998; 102:1358-1363. [PubMed: 9832569]

8. Freeman JM, Vining EP, Kossoff EH, et al. A blinded, crossover study of the efficacy of the ketogenic diet. Epilepsia. 2009; 50:322-325. [PubMed: 18717710]

9. Bough KJ, Wetherington J, Hassel B, et al. Mitochondrial biogenesis in the anticonvulsant mechanism of the ketogenic diet. Ann Neurol. 2006; 60:223-235. [PubMed: 16807920]

10. Kang HC, Lee YM, Kim HD, et al. Safe and effective use of the ketogenic diet in children with epilepsy and mitochondrial respiratory chain complex defects. Epilepsia. 2007; 48:82-88. [PubMed: 17241212]

11••. Haas RH. The evidence basis for coenzyme Q therapy in oxidative phosphorylation disease. Mitochondrion. 2007; 7(Suppl):S136-S145. Dr. Haas gives an in-depth review of CoQ10 chemistry, biochemistry, pharmacokinetics, and the growing evidence base for this medication. [PubMed: 17485245]

12. Quinzii C, Naini A, Salviati L, et al. A mutation in para-hydroxybenzoate-polyprenyl transferase (COQ2) causes primary coenzyme Q10 deficiency. Am J Hum Genet. 2006; 78:345-349. [PubMed: 16400613]

13. Lopez LC, Schuelke M, Quinzii CM, et al. Leigh syndrome with nephropathy and CoQ10 deficiency due to decaprenyl diphosphate synthase subunit 2 (PDSS2) mutations. Am J Hum Genet. 2006; 79:1125-1129. [PubMed: 17186472]

14. Mollet J, Giurgea I, Schlemmer D, et al. Prenyldiphosphate synthase, subunit 1 (PDSS1) and OHbenzoate polyprenyl-transferase (COQ2) mutations in ubiquinone deficiency and oxidative phosphorylation disorders. J Clin Invest. 2007; 117:765-772. [PubMed: 17332895]

15. Lagier-Tourenne C, Tazir M, Lopez LC, et al. ADCK3, an ancestral kinase, is mutated in a form of recessive ataxia associated with coenzyme Q10 deficiency. Am J Hum Genet. 2008; 82:661-672. [PubMed: 18319074]

16. Shults CW, Flint Beal M, Song D, et al. Pilot trial of high dosages of coenzyme Q10 in patients with Parkinson's disease. Exp Neurol. 2004; 188:491-494. [PubMed: 15246848]

17. Bhagavan HN, Chopra RK. Plasma coenzyme Q10 response to oral ingestion of coenzyme Q10 formulations. Mitochondrion. 2007; 7(Suppl):S78-S88. [PubMed: 17482886] 
18. Linnane AW, Kios M, Vitetta L. Coenzyme Q(10)—its role as a prooxidant in the formation of superoxide anion/hydrogen peroxide and the regulation of the metabolome. Mitochondrion. 2007; 7(Suppl):S51-S61. [PubMed: 17482887]

19••. Olsen RK, Olpin SE, Andresen BS, et al. ETFDH mutations as a major cause of ribof avinresponsive multiple acyl-CoA dehydrogenation deficiency. Brain. 2007; 130:2045-2054. This article reviews a genetically proven metabolic disorder leading to mitochondrial dysfunction and its improvement when a simple vitamin is provided as therapy. [PubMed: 17584774]

20. Bugiani M, Lamantea E, Invernizzi F, et al. Effects of ribof avin in children with complex II deficiency. Brain Dev. 2006; 28:576-581. [PubMed: 16737791]

21. Tarnopolsky MA, Raha S. Mitochondrial myopathies: diagnosis, exercise intolerance, and treatment options. Med Sci Sports Exerc. 2005; 37:2086-2093. [PubMed: 16331134]

22. Bernsen PL, Gabreels FJ, Ruitenbeek W, et al. Treatment of complex I deficiency with ribof avin. J Neurol Sci. 1993; 118:181-187. [PubMed: 8229067]

23. Tarnopolsky MA, Parise G. Direct measurement of high-energy phosphate compounds in patients with neuromuscular disease. Muscle Nerve. 1999; 22:1228-1233. [PubMed: 10454718]

24. Moroni I, Bugiani M, Bizzi A, et al. Cerebral white matter involvement in children with mitochondrial encephalopathies. Neuropediatrics. 2002; 33:79-85. [PubMed: 12075488]

25. Tarnopolsky MA, Roy BD, MacDonald JR. A randomized, controlled trial of creatine monohydrate in patients with mitochondrial cytopathies. Muscle Nerve. 1997; 20:1502-1509. [PubMed: 9390662]

26. Borchert A, Wilichowski E, Hanefeld F. Supplementation with creatine monohydrate in children with mitochondrial encephalomyopathies. Muscle Nerve. 1999; 22:1299-1300. [PubMed: 10454733]

27. Koga Y, Ishibashi M, Ueki I, et al. Effects of L-arginine on the acute phase of strokes in three patients with MELAS. Neurology. 2002; 58:827-828. [PubMed: 11889254]

28. Koga Y, Akita Y, Nishioka J, et al. L-arginine improves the symptoms of strokelike episodes in MELAS. Neurology. 2005; 64:710-712. [PubMed: 15728297]

29. Tein I. Carnitine transport: pathophysiology and metabolism of known molecular defects. J Inherit Metab Dis. 2003; 26:147-169. [PubMed: 12889657]

30••. DiMauro S, Hirano M, Schon EA. Approaches to the treatment of mitochondrial diseases. Muscle Nerve. 2006; 34:265-283. This comprehensive review by the Columbia group presents treatment options for mitochondrial myopathy patients. [PubMed: 16810684]

31. Marriage BJ, Clandinin MT, Macdonald IM, et al. Cofactor treatment improves ATP synthetic capacity in patients with oxidative phosphorylation disorders. Mol Genet Metab. 2004; 81:263272. [PubMed: 15059613]

32. Bain MA, Faull R, Fornasini G, et al. Accumulation of trimethylamine and trimethylamine-Noxide in end-stage renal disease patients undergoing haemodialysis. Nephrol Dial Transplant. 2006; 21:1300-1304. [PubMed: 16401621]

33. Ramaekers VT, Weis J, Sequeira JM, et al. Mitochondrial complex I encephalomyopathy and cerebral 5-methyltetra-hydrofolate deficiency. Neuropediatrics. 2007; 38:184-187. [PubMed: 18058625]

34. Garcia-Cazorla A, Serrano M, Perez-Duenas B, et al. Secondary abnormalities of neurotransmitters in infants with neurological disorders. Dev Med Child Neurol. 2007; 49:740-744. [PubMed: 17880642]

35. Stacpoole PW, Kerr DS, Barnes C, et al. Controlled clinical trial of dichloroacetate for treatment of congenital lactic acidosis in children. Pediatrics. 2006; 117:1519-1531. [PubMed: 16651305]

36. Barshop BA, Naviaux RK, McGowan KA, et al. Chronic treatment of mitochondrial disease patients with dichloroacetate. Mol Genet Metab. 2004; 83:138-149. [PubMed: 15464428]

37. Kaufmann P, Engelstad K, Wei Y, et al. Dichloroacetate causes toxic neuropathy in MELAS: a randomized, controlled clinical trial. Neurology. 2006; 66:324-330. [PubMed: 16476929]

38. Prietsch V, Lindner M, Zschocke J, et al. Emergency management of inherited metabolic diseases. J Inherit Metab Dis. 2002; 25:531-546. [PubMed: 12638937]

39. Morgan PG, Hoppel CL, Sedensky MM. Mitochondrial defects and anesthetic sensitivity. Anesthesiology. 2002; 96:1268-1270. [PubMed: 11981173] 
40. Arthur TM, Saneto RP, de Menezes MS, et al. Vagus nerve stimulation in children with mitochondrial electron transport chain deficiencies. Mitochondrion. 2007; 7:279-283. [PubMed: 17513178]

41. Dimmock DP, Dunn JK, Feigenbaum A, et al. Abnormal neurological features predict poor survival and should preclude liver transplantation in patients with deoxyguanosine kinase deficiency. Liver Transpl. 2008; 14:1480-1485. [PubMed: 18825706]

42. Hirano M, Marti R, Casali C, et al. Allogeneic stem cell transplantation corrects biochemical derangements in MNGIE. Neurology. 2006; 67:1458-1460. [PubMed: 16971696]

43. Sinnathuray AR, Raut V, Awa A, et al. A review of cochlear implantation in mitochondrial sensorineural hearing loss. Otol Neurotol. 2003; 24:418-426. [PubMed: 12806294]

44. Tono T, Ushisako Y, Kiyomizu K, et al. Cochlear implantation in a patient with profound hearing loss with the A1555G mitochondrial mutation. Am J Otol. 1998; 19:754-757. [PubMed: 9831149]

45. Spencer CT, Byrne BJ, Gewitz MH, et al. Ventricular arrhythmia in the X-linked cardiomyopathy Barth syndrome. Pediatr Cardiol. 2005; 26:632-637. [PubMed: 16235007]

46. Murphy RT, Mogensen J, McGarry K, et al. Adenosine monophosphate-activated protein kinase disease mimicks hypertrophic cardiomyopathy and Wolff-Parkinson-White syndrome: natural history. J Am Coll Cardiol. 2005; 45:922-930. [PubMed: 15766830]

47. Sanders GD, Hlatky MA, Owens DK. Cost-effectiveness of implantable cardioverter-defibrillators. N Engl J Med. 2005; 353:1471-1480. [PubMed: 16207849]

48. Tarnopolsky MA. Mitochondrial DNA shifting in older adults following resistance exercise training. Appl Physiol Nutr Metab. 2009; 34:348-354. [PubMed: 19448697]

49. Taivassalo T, Haller RG. Exercise and training in mitochondrial myopathies. Med Sci Sports Exerc. 2005; 37:2094-2101. [PubMed: 16331135]

50. Jeppesen TD, Schwartz M, Olsen DB, et al. Aerobic training is safe and improves exercise capacity in patients with mitochondrial myopathy. Brain. 2006; 129:3402-3412. This report shows that exercise is safe for patients with mitochondrial myopathy and is a means to improve mitochondrial functioning. [PubMed: 16815877]

51. Sterba JA, Rogers BT, France AP, et al. Horseback riding in children with cerebral palsy: effect on gross motor function. Dev Med Child Neurol. 2002; 44:301-308. [PubMed: 12033715]

52. Puigserver P, Spiegelman BM. Peroxisome proliferator- activated receptor-gamma coactivator 1 alpha (PGC-1 alpha): transcriptional coactivator and metabolic regulator. Endocr Rev. 2003; 24:78-90. [PubMed: 12588810]

53. Wenz T, Diaz F, Spiegelman BM, et al. Activation of the PPAR/PGC-1alpha pathway prevents a bioenergetic def cit and effectively improves a mitochondrial myopathy phenotype. Cell Metab. 2008; 8:249-256. [PubMed: 18762025]

54. Saiko P, Szakmary A, Jaeger W, Szekeres T. Resveratrol and its analogs: defense against cancer, coronary disease and neurodegenerative maladies or just a fad? Mutat Res. 2008; 658:68-94. [PubMed: 17890139]

55. Silva MF, Aires CC, Luis PB, et al. Valproic acid metabolism and its effects on mitochondrial fatty acid oxidation: a review. J Inherit Metab Dis. 2008; 31:205-216. [PubMed: 18392741]

56. Dalakas MC. Peripheral neuropathy and antiretroviral drugs. J Peripher Nerv Syst. 2001; 6:14-20. [PubMed: 11293802]

57. Scruggs ER, Dirks Naylor AJ. Mechanisms of zidovudine-induced mitochondrial toxicity and myopathy. Pharmacology. 2008; 82:83-88. [PubMed: 18504416]

58. Pinti M, Salomoni P, Cossarizza A. Anti-HIV drugs and the mitochondria. Biochim Biophys Acta. 2006; 1757:700-707. [PubMed: 16782042]

59•. Littarru GP, Langsjoen P. Coenzyme Q10 and statins: biochemical and clinical implications. Mitochondrion. 2007; 7(Suppl):S168-174. This article provides an example of how commonly used medications can lead to mitochondrial dysfunction. [PubMed: 17482884]

60. Sirvent P, Mercier J, Lacampagne A. New insights into mechanisms of statin-associated myotoxicity. Curr Opin Pharmacol. 2008; 8:333-338. [PubMed: 18243052]

61. Wagner BK, Kitami T, Gilbert TJ, et al. Large-scale chemical dissection of mitochondrial function. Nat Biotechnol. 2008; 26:343-351. [PubMed: 18297058] 
62. Bindu LH, Reddy PP. Genetics of aminoglycoside-induced and prelingual non-syndromic mitochondrial hearing impairment: a review. Int J Audiol. 2008; 47:702-707. [PubMed: 19031229]

63. Fischel-Ghodsian N. Genetic factors in aminoglycoside toxicity. Ann NY Acad Sci. 1999; 884:99109. [PubMed: 10842587]

64. Kovacic P, Pozos RS, Somanathan R, et al. Mechanism of mitochondrial uncouplers, inhibitors, and toxins: focus on electron transfer, free radicals, and structure-activity relationships. Curr Med Chem. 2005; 12:2601-2623. [PubMed: 16248817]

65. Spiller HA, Sawyer TS. Toxicology of oral antidiabetic medications. Am J Health Syst Pharm. 2006; 63:929-938. [PubMed: 16675650] 
Table 1

Drugs with reported mitochondrial toxicity

\begin{tabular}{|c|c|c|}
\hline Medication & Symptoms & Mechanism \\
\hline Valproic acid [55] & $\begin{array}{l}\text { Hepatopathy; infrequently direct } \\
\text { encephalopathy }\end{array}$ & $\begin{array}{l}\text { Inhibition of fatty acid oxidation, the citric acid cycle, } \\
\text { and oxidative phosphorylation; carnitine depletion; } \\
\text { complex IV inhibition (contraindicated in } \\
\text { mitochondria depletion syndromes) }\end{array}$ \\
\hline Antiretrovirals [56-58] & $\begin{array}{l}\text { Peripheral neuropathy, liver dysfunction, } \\
\text { myopathy }\end{array}$ & $\begin{array}{l}\text { Impairment of mtDNA replication causing mtDNA } \\
\text { depletion; carnitine defi ciency, lactic acidosis, } \\
\text { lipodystrophy [59] }\end{array}$ \\
\hline Statins $[59 \bullet, 60,61]$ & Myopathy & $\begin{array}{l}\text { Multiple postulated effects, including CoQ10 } \\
\text { depletion }\end{array}$ \\
\hline Aspirin & Reye syndrome & $\begin{array}{l}\text { Inhibition and uncoupling of oxidative } \\
\text { phosphorylation }\end{array}$ \\
\hline Aminoglycoside antibiotics $[62,63]$ & $\begin{array}{l}\text { Hearing loss, cardiac toxicity, renal } \\
\text { toxicity }\end{array}$ & Impaired mtDNA translation \\
\hline $\begin{array}{l}\text { Aminoglycoside and platinum } \\
\text { chemotherapeutics [64] }\end{array}$ & $\begin{array}{l}\text { Hearing loss, cardiac toxicity, renal } \\
\text { toxicity }\end{array}$ & Impaired mtDNA translation \\
\hline Acetaminophen & Hepatopathy & Oxidative stress \\
\hline Metformin [65] & Lactic acidosis & $\begin{array}{l}\text { Inhibition of oxidative phosphorylation, enhanced } \\
\text { glycolysis }\end{array}$ \\
\hline Beta-blockers [61] & Reduced exercise tolerance & Oxidative stress \\
\hline Steroids & $\begin{array}{l}\text { Reports of deterioration in Kearns-Sayre } \\
\text { syndrome }\end{array}$ & Unknown \\
\hline
\end{tabular}

CoQ10—coenzyme Q10; mtDNA—mitochondrial DNA. 

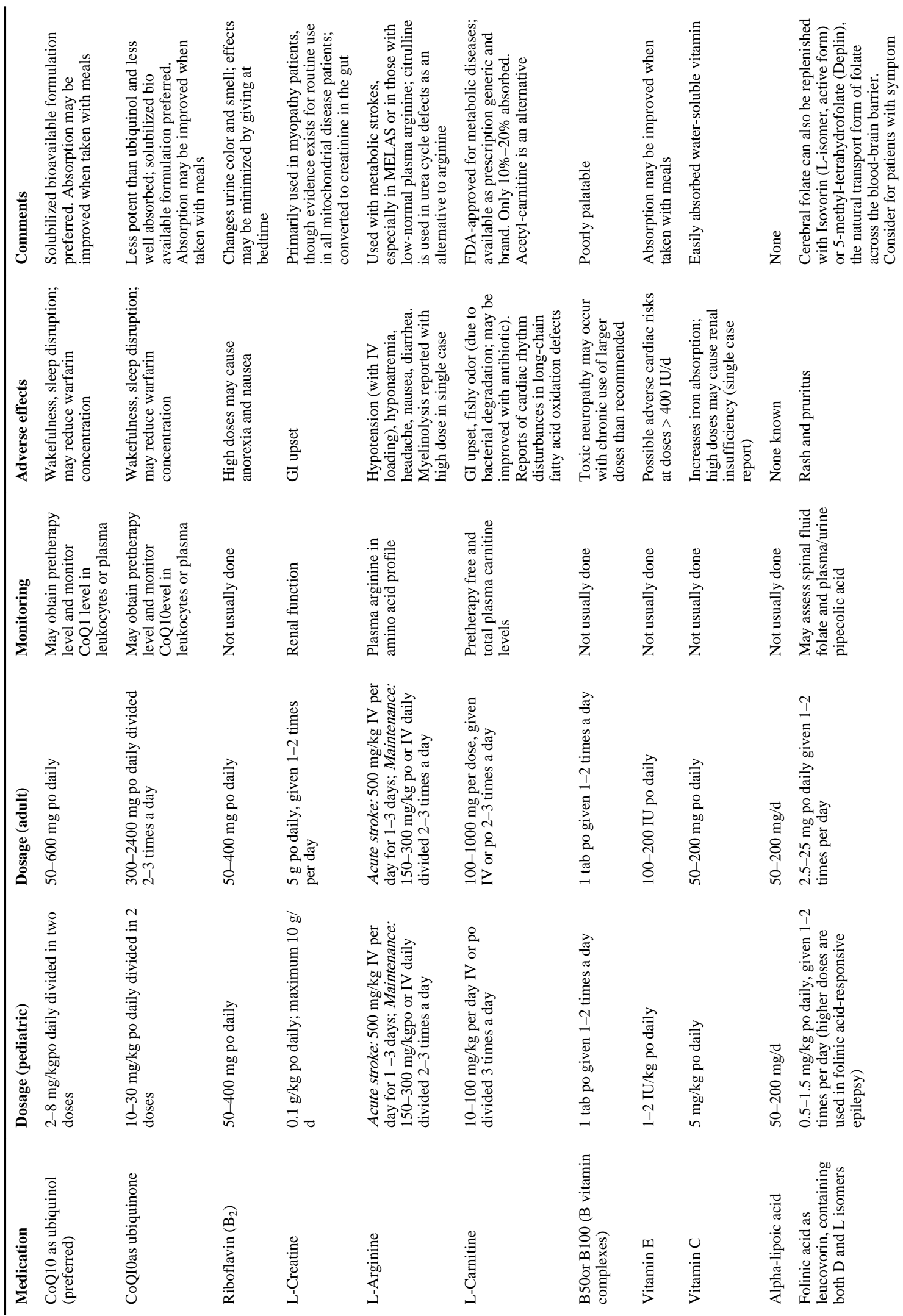


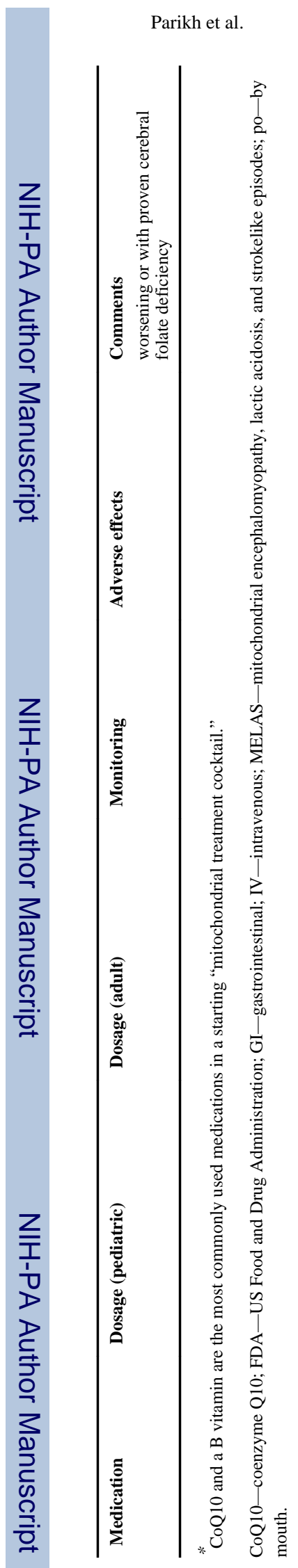

Curr Treat Options Neurol. Author manuscript; available in PMC 2013 January 31. 